\title{
Potential self-medication by brown titi monkeys, Plecturocebus brunneus, in an urban fragment forest in the Brazilian Amazon
}

\author{
Brenda Letícia Pereira Oliveira ${ }^{1}$, João Pedro Souza-Alves ${ }^{2,4}$, and Marcela Alvares Oliveira ${ }^{1,3}$ \\ ${ }^{1}$ Curso de Ciências Biológicas, Centro Universitário Aparício Carvalho, Porto Velho, \\ Rondônia,76811-678, Brazil \\ ${ }^{2}$ Programa de Pós-Graduação em Biologia Animal, Universidade Federal de Pernambuco, \\ Recife, 50670-901, Brazil \\ ${ }^{3}$ Programa de Pós-Graduação em Biodiversidade e Biotecnologia da Amazônia Legal, Universidade Federal de \\ Rondônia Rondônia, Porto Velho, 76812-245, Brazil \\ ${ }^{4}$ Laboratório de Ecologia, Comportamento e Conservação (LECC), Departamento de Zoologia, Universidade \\ Federal de Pernambuco, Recife, 50670-901, Brazil
}

Correspondence: Marcela Alvares Oliveira (marcela.mugrabe@gmail.com)

Received: 3 October 2020 - Revised: 27 October 2020 - Accepted: 27 October 2020 - Published: 15 December 2020

\begin{abstract}
In this study, we report fur-rubbing behavior of brown titi monkeys, Plecturocebus brunneus, using chewed leaves from (Fabaceae) and Piper tuberculatum (Piperaceae). These reports were obtained during systematic monitoring of titi monkeys from May until December 2019 (218 h) in an urban fragment forest in the Brazilian Amazon. Both plant species contain chemical substances in their leaves that potentially repel ectoparasites. The genus Piper is known for its repelling action due to the presence of amides, alkaloids and benzoic acid. The presence of dogs, cats and human settlements may contribute to an increase of ectoparasites, making a potential self-medication function of fur rubbing in this primate species plausible.
\end{abstract}

\section{Introduction}

The fur-rubbing or self-anointing behavior from different plant parts and insects is relatively well documented in wild and captive Neotropical primates. Overall, this behavior may be associated with several functions such as repelling or killing of ectoparasites and microbial pathogens (selfmedication), and scent marking (olfactory communication) (Huffman et al., 2013; Martinez et al., 2019). The chemical substances emerging from plants and arthropods, after being chewed and crushed, act as therapeutic medicine for the species (Huffman, 2011) due to the presence of metabolic compounds with inhibitory action (therapeutic action) on parasites (Peckre et al., 2018).

Several plant genera are frequently used by primates in self-medication events. Capuchins (Cebus capucinus), for example, use Citrus, Sloanea, Clematis and Piper plants (Baker, 1996), captive and wild titi monkeys Piper and Bauhinia (Huashuayo-Llamocca and Heymann, 2017; Souza-Alves et al., 2018, respectively), and spider monkeys (Ateles geoffroyi) Citrus and Zanthoxylum (Campbel, 2000). Arthropods are also used often, as for example millipedes can secrete caustic benzoquinones that work quickly on the fur and body of primates and are repellent to insects (Valderrama et al., 2000). Such actions tend to increase in frequency during the rainy period when the risks of insect bites and infections are higher (Huffman, 1997).

Titi monkeys are small-bodied primates living in family groups consisting of a breeding pair, one juvenile and one offspring (Bicca-Marques and Heymann, 2013). They devote most of their activity time to resting and feed mainly on fleshy fruits (Bicca-Marques and Heymann, 2013). Furrubbing behavior in titi monkeys has been documented and interpreted both as self-medication (Carrillo-Bilbao et al., 2005; Huashuayo-Llamocca and Heymann, 2017) and olfactory communication (Souza-Alves et al., 2018). The 
brown titi monkey, Plecturocebus brunneus, is distributed across the tropical forest from Bolivia, Brazil and Peru (Veiga et al., 2008). In Brazil, this species is endemic in the state of Rondônia, inhabiting continuous areas of Amazonian forests as well as urban forest fragments (Ferrari et al., 2000), where it can be found in small fragments $\left(<1 \mathrm{~km}^{2}\right)$ (Medeiro et al., 2019). Most research on these Brazilian populations of $P$. brunneus focused on their geographical distribution and general behavioral activities (Ferrari et al., 2000; SouzaAlves et al., 2019). Consequently, reporting rare behaviors such as fur rubbing increases our understanding of the socioecology of this species. Here, we report fur-rubbing behavior in one group of $P$. brunneus in an urban forest fragment in the South-Occidental Amazon.

\section{Methods}

Our reports were performed during systematic monitoring conducted in an urban fragment of the Amazon in the municipality of Porto Velho $\left(8^{\circ} 44^{\prime} 3336^{\prime \prime} 5 \mathrm{~S}, 63^{\circ} 52^{\prime} 1850^{\prime \prime} 0\right)$, northern Brazil. The urban forest is categorized as a protected area according to Brazilian Forest Law (decree number 12.651/2012). Although the area is legally protected it suffers negative impact and size reduction due to anthropogenic disturbances. Adjoining the area is a car wash and houses without a sewage treatment network, acting as constant sources of solid waste and water, a common condition in different urban fragments of the municipality (Moreira and Oliveira, 2019). Currently, the forest fragment is comprised of ca. $2000 \mathrm{~km}^{2}$ composed of secondary vegetation with a high richness of exotic plant species such as Musa paradisiaca (banana) and Persea americana (avocado). The mean annual rainfall varies between 2000 and $2300 \mathrm{~mm}$, with high temperatures from 24 to $27^{\circ} \mathrm{C}$ across the year. The dry season is short, encompassing 2 and 3 months (July-August), reaching maximum temperatures up to $37^{\circ} \mathrm{C}$ (Mendonça and Danni-Oliveira, 2014).

The titi monkey group was monitored from May to December 2019 on $3 \mathrm{~d}$ per month between 08:00 and 15:00 AMT (Amazon time, UTC-4) yielding a total of 218 observation hours across $35 \mathrm{~d}$. During this period the study group was composed of three individuals: a breeding pair and a juvenile of unidentified sex (Fig. 1). Fur-rubbing behaviors were recorded ad libitum (Altmann, 1974). When possible, the plant species and specific parts used were identified.

\section{Results}

Fur-rubbing behavior was reported on a total of 27 occasions. On 12 occasions ( 8 times by the adult male, 4 times by the adult female) titi monkeys used Senna obtusifolia (Fabaceae) leaves, and on the remaining 15 occasions (10 times by the adult male, 5 times by the adult female) they used Piper tuberculatum (Piperaceae) leaves. Using S. obstusifolia leaves, individuals picked the leaves from the tree, chewed them and subsequently rubbed the chewed leaves on their chest. This behavior was usually seen when the individuals were alone on a tree branch and during the morning two to three times. At no time did titi monkeys rub their bodies on tree branches or against the body of group members after chewing the leaves.

During fur-rubbing behavior using $P$. tuberculatum leaves, all members of the study group were present. Individuals removed leaves, chewed them for a short period (2-5s) and rubbed the chewed leaves on both hands. It was not observed whether the individuals rubbed their hands subsequently on tree branches or other individuals. All these events were observed in the morning between 08:00 and 10:00 AMT (Amazon time, UTC-4).

\section{Discussion}

From anecdotal reports, we described the fur-rubbing behavior in a poorly studied species of titi monkey, Plecturocebus brunneus, in an urban Amazonian fragment forest in Brazil. Owing to the chemical characteristics of the plant species, it seems likely that this behavior is a form of selfmedication. Senna obtusifolia is an invasive species in Brazil with possible origin in the Caribbean and the northern region of South America (Randell, 1995). It has different allelopathic properties (e.g., Abdullahi et al., 2017), and among humans, it is used in traditional medicine as treatment for gingivitis, urinary tract infections, diarrhea, fever and cough (Doughari et al., 2008; Mahwasanel et al., 2013). Toxicity of the leaf extract of Senna presents an antimicrobial action with the presence of saponins, tannins, alkaloids and flavonoids (Doughari et al., 2008). Piperaceae is considered a pantropical family, with species being found from Mexico to southeastern Argentina (Figueiredo and Sazima, 2000). Medical use includes several treatments such as recovery from childbirth, toothache, stomach pain, colds and symptoms of erysipelas - a bacterial infection in the upper layer of the skin (Martínez-Bautista et al., 2019). Piper tuberculatum has proven to act in laboratory tests as an anti-drug (Silva Lima et al., 2014), anti-tumor (Bezerra et al., 2015), anti-leishmania (Ferreira et al., 2010) and as an insecticide (Bazán-Calderón et al., 2011). This genus presents various biological compounds such as amides, alkaloids and benzoic acid (Parmar et al., 1997). P. tuberculatum leaves are composed of 4,5-dihydropiperlonguminine, piperlongumine, 4,5-dihydropiperine and piperine (Scott et al., 2002). These chemical substances demonstrate a strong insecticide activity (Bazán-Calderón et al., 2011).

Although leaves of Senna obtusifolia present chemical compounds with the ability to act on different diseases, their role in self-medication of $P$. brunneus remains unclear. In contrast, behavior similar to the one reported here has been observed previously in titi monkeys using different 


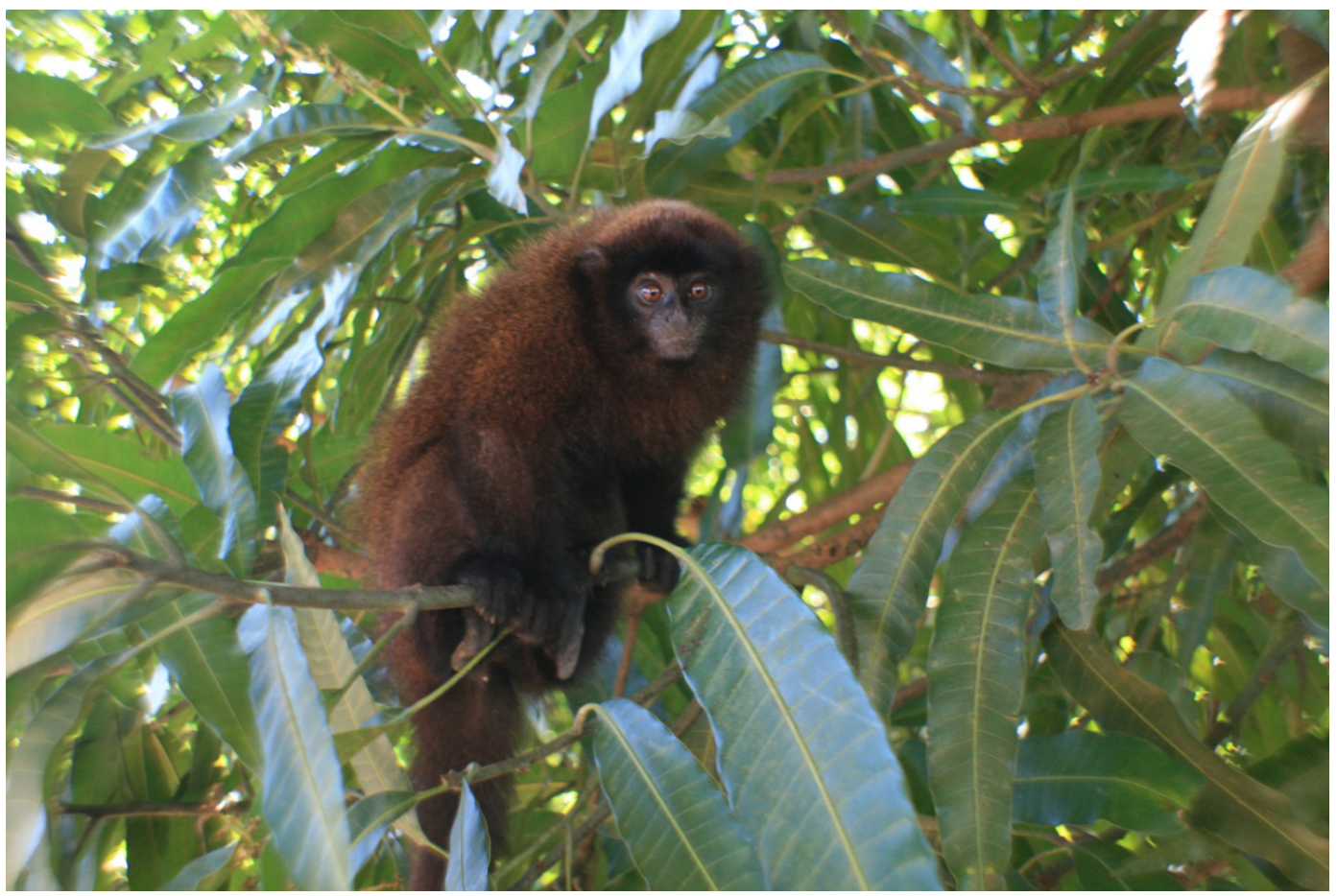

Figure 1. Juvenile member of brown titi monkey (Plecturocebus brunneus) study group in the urban fragment forest. Photo: Rallison Viamonte.

Piper species (Huashuayo-Llamocca and Heymann, 2017; Martínez et al., 2019). Due to the insecticidal properties of different species of the genus Piper and the characteristics of the method of use, both studies concluded this behavior to be related to self-medication in order to repel insects. As such, it seems reasonably likely that the events recorded for $P$. brunneus in this study represent self-medication behavior.

Further evidence is needed to determine the direct stimulus for fur rubbing at this site. Additionally, the characteristics of the site, such as the presence of housing (including humans, dogs and cats) and the constant disposal of water can favor the reproduction of ectoparasites (Solórzano-Garcia and Pérez-Ponce de Léon, 2018), which may lead to individuals of $P$. brunneus to performing fur-rubbing self-medication behavior. Martínez et al. (2019) point out that the long and dense fur of the titi monkeys acts as a barrier to the action of insects, but the hands, neck and chest would be devoid of this type of protection. As titi monkeys in this study used the products of chewing on the hands and breast region and both plants have different allelopathic functions, this behavior reinforces the possibility of using these plants as a repellent from parasites or relief from their irritative effect.

Anecdotal evidence often points to characteristics that can be later confirmed through more rigorous scientific analysis (Browning, 2017). In this context, it is plausible to speculate that the use of the leaves of S. obtusifolia and P. tuberculatum used for the P. brunneus is associated with self-medication behavior (Huffman, 1997).
Data availability. There are no further data apart from the observations reported in the paper.

Author contributions. BLPO and MAO formulated the overarching research goals and aims. BLPO conducted the research and investigation process, specifically the data collection. JPSA and MAO prepared and created the published work, specifically the writing of the initial draft (including substantive translation).

Competing interests. The authors declare that they have no conflict of interest.

Acknowledgements. We are grateful to Elsie Guimarães from Jardim Botânico do Rio de Janeiro for the identification of Piper tuberculatum. João Pedro Souza-Alves is supported by FACEPE (grant no. BFP-0149-2.05/19). We are grateful to Michael Huffman and one anonymous referee for their valuable contributions to our manuscript.

Review statement. This paper was edited by Julia Ostner and reviewed by Michael Huffman and one anonymous referee. 


\section{References}

Abdullahi, M. Y., Musa, D. D., and Oti, E. C.: Allelopathic activity of aqueous leaf extract of Eucalyptus camaldulensis on the germintaion and growth of cowpea and Senna obtusifolia, IJCSS, 1, 41-46, 2017.

Altmann, J.: Observational study of behavior: sampling methods, Behaviour, 49, 227-267, https://doi.org/10.1163/156853974x00534, 1974.

Baker, M.: Fur rubbing: use of medicinal plants by capuchin monkeys (Cebus capucinus), Am. J. Primatol., 38, 263-270, https://doi.org/10.1002/(SICI)1098-2345(1996)38:3<263::AIDAJP5>3.0.CO;2-X, 1996.

Bazán-Calderón, J., Ventura-Flores, R., Kato, M. J., Rojas-Idrogo, C., and Delgado-Paredes, G. E.: Actividad insecticida de Piper tuberculatum Jacq. sobre Aedes aegypti L. (Diptera: Culicidae) y Anopheles pseudopunctipennis Tehobal (Diptera: Culicidae), Anales de Biología, 33, 135-147, 2011.

Bezerra, D. P., Ferreira, P. M. P., Machado, C. M. L., de Aquino, N. C., Silveira, E. R., Chammas, R., and Pessoa, C.: Antitumour efficacy of Piper tuberculatum and piplartine based on the hollow fiber assay, Planta Med., 81, 15-19, https://doi.org/10.1055/s0034-1383363, 2015.

Bicca-Marques, J. C. and Heymann, E. W.: Ecology and behavior of titi monkeys (genus Callicebus), in: Evolutionary biology and conservation of titis, sakis and uacaris, edited by: Veiga, L. M., Barnett, A. A., Ferrari, S. F., and Norconk M. A., Cambridge University Press, Cambridge, Chap. 17, 196-207, https://doi.org/10.1017/cbo9781139034210.023, 2013.

Browning, H.: Anecdotes can be evidence too, Animal Sentience, 16, 1-6, 2017.

Campbell, C. J.: Fur rubbing behavior in free-ranging blackhanded spider monkeys (Ateles geoffroyi) in Panama, Am. J. Primatol., 51, 205-208, https://doi.org/10.1002/10982345(200007)51:3<205::AID-AJP5>3.0.CO;2-L, 2000.

Carrillo-Bilbao, G., Di Fiore, A., and Fernández-Duque, E.: Dieta, forrajeo y presupuesto de tiempo en cotoncillos (Callicebus discolor) del Parque Nacional Yasuní en la Amazonia Ecuatoriana, Neotropical Primates, 13, 7-11, https://doi.org/10.1896/14134705.13.2.7, 2005.

Doughari, J. H., El-Mahmood, A. M., and Tyoyina, I.: Antimicrobial activity of leaf extracts of Senna obtusifolia (L), Afr. J. Pharm. Pharmaco., 2, 7-13, 2008.

Ferrari, S. F., Iwanaga, S., Messias, M. R., Ramos, E. M., Ramos, P. C. S., da Cruz Neto, E. H., and Coutinho, P. E. G.: Titi monkeys (Callicebus spp., Atelidae: Platyrrhini) in the Brazilian state of Rondônia, Primates, 41, 229, https://doi.org/10.1007/BF02557805, 2000.

Ferreira, M. G. P., Kayano, A. M., Silva-Jardim, I., da Silva, T. O., Zuliani, J. P., Facundo, V. A., Calderon, L. A., Almeida-e-Silva, A., Ciancaglini, P., and Stábeli, R. G.: Antileishmanial activity of 3-(3, 4, 5-trimethoxyphenyl) propanoic acid purified from Amazonian Piper tuberculatum Jacq., Piperaceae, fruits, Rev. Bras. Farmacogn., 20, 1003-1006, https://doi.org/10.1590/S0102-695X2010005000033, 2010.

Figueiredo, A. R. and Sazima, M.: Pollination biology of Piperaceae species in southeastern Brazil, Ann. Bot., 85, 455-460, https://doi.org/10.1006/anbo.1999.1087, 2000.
Huashuayo-Llamocca, R. and Heymann, E. W.: Fur-rubbing with Piper leaves in the San Martín titi monkey, Callicebus oenanthe, Primate Biol., 4, 127-130, https://doi.org/10.5194/pb-4-1272017, 2017.

Huffman, M. A.: Current evidence for self-medication in primates: $m$ multidisciplinary perspective, Am. J. Phys. Anthropol., 104, 171-200, https://doi.org/10.1002/(SICI)10968644(1997)25+<171::AID-AJPA7>3.0.CO;2-7, 1997.

Huffman, M. A.: Primate self-medication, in: Primates in Perspectiv, 2nd edn., edited by: Campbell, C. J., Fuentes, A., MacKinnon, K. C., Bearder, S. K., and Stumpf, R. M., Oxford University Press, Oxford, 563-575, 2011.

Huffman, M. A., Nakagawa, N., Go, Y., Imai, H., and Tomonaga, M.: Primate self-medication and the treatment of parasite infection, in: Monkeys, Apes, and Humans, Springer, Tokyo, 13-23, https://doi.org/10.1007/978-4-431-54153-0_2, 2013.

Mahwasane, S. T., Middleton, L., and Boaduo, N.: An ethnobotanical survey of indigenous knowledge on medicinal plants used by the traditional healers of the Lwamondo area, Limpopo province, South Africa, S. Afr. J. Bot., 88, 69-75, https://doi.org/10.1016/j.sajb.2013.05.004, 2013.

Martínez-Bautista, B. G., Bernal-Ramírez, L. A., BravoAvilez, D., Samain, M. S., Amezcua, J. M. R., and Rendón-Aguilar, B.: Traditional Uses of the Family Piperaceae in Oaxaca, Mexico, Trop. Conserv. Sci., 12, 1-22, https://doi.org/10.1177/1940082919879315, 2019.

Medeiro, A. Z., Araújo, L. S., and Oliveira, M. A.: Riqueza de mamíferos de médio e grande porte em Área de Preservação Permanente do distrito de Jaci Paraná, Rondônia, RBCA, 8, 1-8, 2019.

Mendonça, F. and Danni-Oliveira, I. M. (Eds.): Climatologia: Noções básicas e climas do Brasil, Oficina de Textos, São Paulo, 206 pp., 2014.

Moreira, B. R. and Oliveira, M. A.: Percepção ambiental de moradores do entorno de Áreas de Preservação Permanente em Porto Velho, Rondônia, SAJEBTT, 6, 213-226, 2019.

Parmar, V. S., Jain, S. C., Bisht, K. S., Jain, R., Taneja, P., Jha, A., Tyagi, O. M., Prasad, A. K., Wengel, J., Olsen, C. E., and Boll, P. M.: Phytochemistry of the genus Piper, Phytochemistry, 46, 597-673, https://doi.org/10.1016/S0031-9422(97)00328-2, 1997.

Peckre, L. R., Defolie, C., Kappeler, P. M., and Fichtel, C.: Potential self medication using millipede secretions in red fronted lemurs: combining anointment and ingestion for a joint action against gastrointestinal parasites?, Primates, 59, 483-494, https://doi.org/10.1007/s10329-018-0674-7, 2018.

Randell, B. R.: Taxonomy and evolution of Senna obtusifolia and S. tora, Journal of the Adelaide Botanic Gardens, 16, 55-58, 1995.

Scott, I. M., Puniani, E., Durst, T., Phelps, D., Merali, S., Assabgui, R. A., Sánchez-Vindas, P., Poveda, L., Philogène, B. R. and Arnason, J. T.: Insecticidal activity of Piper tuberculatum Jacq. extracts: synergistic interaction of piperamide, Agric. For. Entomol., 4, 137-144, https://doi.org/10.1046/j.14619563.2002.00137.x, 2002.

Silva Lima, A., Filho, J. G. N, S., Pereira, S. G., Guillon, G. M. S. P., da Silva Santos, L., and Costa, L. M.: Acaricide activity of different extracts from Piper tuberculatum fruits 
against Rhipicephalus microplus, Parasitol. Res., 113, 107-112, https://doi.org/10.1007/s00436-013-3632-8, 2014.

Solórzano-García, B. and Pérez-Ponce de León, G.: Parasites of neotropical primates: a review, Int. J. Primatol., 39, 155-182, https://doi.org/10.1007/s10764-018-0031-0, 2018.

Souza-Alves, J. P., Albuquerque, N. M., Vinhas, L., Cardoso, T. S., Beltrão-Mendes, R., and Jerusalinsky, L.: Self-anointing behaviour in captive titi monkeys (Callicebus spp.), Primate Biol., 5, 1-5, https://doi.org/10.5194/pb-5-1-2018, 2018.

Souza-Alves, J. P., Mourthe, I., Hilário, R. R., Bicca-Marques, J. C., Rehg, J., Gestich, C. C., Acero-Murcia, A. C., Adret, P., Aquino, R., Berthet, M., Bowler, M., Calouro, A. M., Canale, G. R., Cardoso, N. de A., Caselli, C. B., Cäsar, C., Chagas, R. R. D., Clyvia, A., Corsini, C. F., Defler, T., DeLuycker, A., Di Fiore, A., Dingess, K., Erkenswick, G., Ferreira, M. A., Fernandez-Duque, E., Ferrari, S. F., Fontes, I. P., Gomes, J. D., Gonçalves, F. P. R., Guerra, M., Haugaasen, T., Heiduck, S., Heymann, E. W., Hodges, S., Huashuayo-Llamocca, R., Jerusalinsky, L., Kasper, C. B., Lawrence, J., Lueffe, T. M., Lopes, K. G. D., Martínez, J., de Melo, F. R., Messias, M. R., Nagy-Reis, M. B., Nole, I., Paciência, F., Palacios, E., Poirier, A., Porfírio, G., Porter, A., Price, E., Printes, R. C., Quintino, E. P., Reis, E. A., Rocha, A., Rodríguez, A., Röhe, F., Rumiz, D., Shanee, S., Santana, M. M., Setz, E. Z. F., de Souza, F. S. C., Spironello, W., Tirado Herrera, E. R., Vinhas, L., Vulinec, K., Wallace, R. B., Watsa, M., Wright, P. C., Young, R. J., and Barnett, A. A.: Terrestrial behavior in titi monkeys (Callicebus, Cheracebus, and Plecturocebus): Potential correlates, patterns, and differences between genera, Int. J. Primatol., 40, 553-572, https://doi.org/10.1007/s10764-019-00105x, 2019.
Valderrama, X., Robinson, J. G., Attygalle, A. B., and Eisner, T.: Seasonal anointment with millipedes in a wild primate: a chemical defense against insects?, J. Chem. Ecol., 26, 2781-2790, https://doi.org/10.1023/A:1026489826714, 2000.

Veiga, M. L., Wallace, B. R., and Ferrari, S. F.: Plecturocebus brunneus, The IUCN Red List of Threatened Species 2008: e.T41558A10479157, available at: https://www.iucnredlist.org/ species/41558/10479157 (last access: 27 July 2020), 2008. 